Classification

Physics Abstracts

$47.10-47.55 \mathrm{~K}-47.80$

\title{
Effect of a plane wall on a sphere moving parallel to it
}

\author{
A. Ambari (*), B. Gauthier Manuel and E. Guyon \\ Laboratoire d'Hydrodynamique et de Mécanique Physique (**), \\ E.S.P.C.I., 10, rue Vauquelin, 75231 Paris Cedex 05, France
}

(Reçu le 22 octobre 1982, révisé le 14 décembre, accepté le 23 décembre 1982)

\begin{abstract}
Résumé. - L'étude de l'effet d'une paroi plane sur la force hydrodynamique exercée sur une sphère en mouvement de translation parallèle, pose des problèmes expérimentaux. Nous présentons ici une méthode originale permettant la mesure de cette force tout en imposant une valeur constante de la distance entre la sphère et la paroi. Les résultats de cette expérience sont confrontés aux théories de O'Neill, de Goldman et al., et de Faxén.
\end{abstract}

\begin{abstract}
The effect of a plane wall on the hydrodynamic force exerted on a sphere moving parallel to it presents experimental difficulties. We describe an original method for the measurement of the force while keeping constant the distance between the wall and the sphere. The results are compared with the theoretical models of O'Neill, of Goldman et al., and of Faxén.
\end{abstract}

The study of hydrodynamic suspensions of spherical objects in fluids is complex [1] : it involves the determination of interparticle interactions which control the particles distribution; this distribution itself determines the dynamical behaviour of each individual particle. A simpler case, more studied theoretically, is that of a fixed bed where the distribution of solid objects is rigidly determined as is the case in the free fall of a pair of spheres of equal radius [2] or in a dense array of particles.

We consider here a particular example of such a distribution, the fall of a sphere S (radius $a$ ) parallel to a vertical plane wall W, in a viscous liquid (inset of Fig. 1). We use an original rheometer, developed in our laboratory [3], which uses a magnetic sphere maintained at a fixed level by levitation in a vertical field $H_{x}$. The horizontal position - in particular the distance $b$ to the vertical wall $\perp$ to $y$ is adjusted by an additional horizontal field $H_{y}(y)$ given by a pair of vertical Helmholtz coils in opposition and giving a constant gradient $\mathrm{d} H_{y} / \mathrm{d} y$. A vertical glass tube containing the viscous liquid is limited to half a cylinder by fitting in it a solid lucite wall $\mathrm{W}$ in a diametral plane. The assembly is moved at a constant vertical velocity $U_{x}$. The Stokes force $F_{x}$ impressed on the sphere, whose fixed position within the half cylinder tube is controlled optically, is detected as an additional current sent, by a feed-back system, to the horizontal coil which produces the

(*) Also Laboratoire de Physique des Liquides et Electrochimie, Université P. et M. Curie, T 22, 4, place Jussieu, 75230 Paris Cedex 05.

$\left({ }^{* *}\right)$ E.R.A. $n^{\circ} 1000$. 


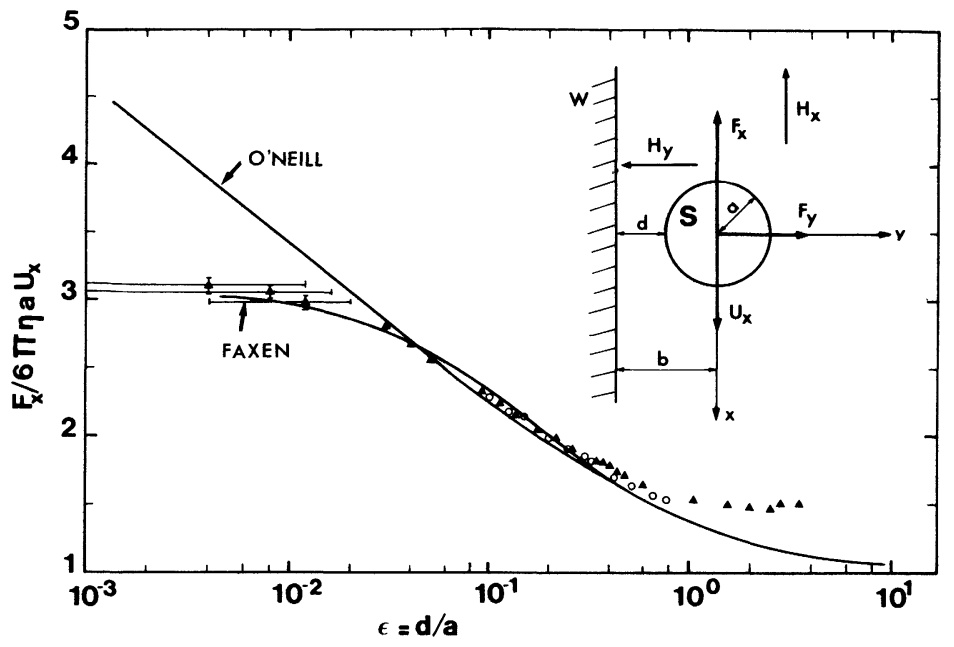

Fig. 1. - The figure gives the value of the vertical Stokes force exerted on a sphere near a wall and normalized to the usual Stokes result far away from it. The distance is measured by the lubrication ratio $\varepsilon=d / a$. The geometry of the sphere rheometer experiment and of the coordinate system is given schematically in figure 1. ( $\triangle$ silicone oil 47 V 100; o silicone oil 47 V 20.)

field $H_{x}$. Thus the experiment leads to the measurement of the variation of the Stokes force $F_{x}$ exerted on a sphere of radius $a$ moving, at a constant velocity $U_{x}$, at a fixed distance $b$ from a wall [4].

This last feature is an original characteristic of the present experiment. In the free fall of a sphere, the presence of a wall gives rise, when $b$ becomes of the order of the radius $a$, to two contributions : i) a decrease of the velocity of translation and a rotation of particles in the case of spherical objects, often interpreted as a slip effect at the walls [5]; ii) when inertial terms become appreciable, a transverse force $F_{y}$ away from the wall $[2,6,11]$. This effect leads to a decrease of concentration near the wall which adds up to a purely steric contribution. The resultant of these two actions is called the $\Sigma$ effect [7-8]. Thus, when the viscosity is measured with two set ups having different characteristic dimensions comparable with $a$, the measurements on suspensions (unlike those on pure Newtonian fluids) will lead to different results.

Lorentz [9] and Stock [10] were the first to solve the effect of a fixed plane wall on a sphere $S$ moving parallel to it (our experimental situation not including the $\Sigma$ effect), the fluid being at rest at infinity and not being submitted to any constraint. But it was Faxén [11] who gave a solution of the problem satisfying Oseen's equation whilst allowing the sphere to rotate. He used a " method of reflections ". With a 4th order expansion (two reflexions on $\mathrm{P}$ and a single one on $\mathrm{S}$ ), he obtained an expression for the hydrodynamic force :

$$
F_{x} /\left(6 \pi \eta a U_{x}\right)=\left[1-\frac{9}{16} k \cdot f\left(\frac{U_{x} b}{2 v}\right)+\frac{k^{3}}{8}-\frac{45 k^{4}}{256}-\frac{k^{5}}{16}\right]^{-1},
$$

$b=d+a$ is the distance of the centre of $\mathrm{S}$ to $\mathrm{W} ; k=a \mid b$.

The numerical values of $f(x)$ and the expression for $F_{y}$, which is directed away from $\mathrm{W}$, are given in reference [11].

In equation (1), the force $E_{x}$ is normalized to the usual Stokes expression obtained far away from the wall $(k \rightarrow 0)$. The expansion is valid when $k$ is small and when the Reynolds number associated with the sphere's radius, $R e=a U_{x} / v$, is also small. 
Faxén also showed that the sphere undergoes a rotation around the $z$ axis with an angular velocity $\omega$ in the direction that it would have if it were in contact with $\mathbf{W}$ and would roll without shearing.

The theoretical results are given in figure 1 in the limit where inertial terms are negligible : $f\left(U_{x} b / 2 v\right) \sim 1$ (which corresponds to the experimental condition). We also give the numerical result of an « exact » calculation by O'Neill [12] done by solving the Stokes equation using bipolar coordinates. However the solution corresponds to the case where the sphere moves parallel to the wall without rotation. This result was confirmed by a calculation $[13,14]$ in the lubrication limit $\varepsilon=d / a \ll 1$.

The experiments were done using a sphere of diameter $2 a=1 \mathrm{~mm}$ and a variable distance to the wall ( $d / a \sim 5 \times 10^{-2}$ to 3 ) (Fig. 2). The accuracy on the measurements of the smallest values

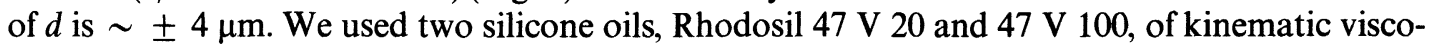
sities $v=0.2$ and $1 \mathrm{~cm}^{2} / \mathrm{s}$ at $20{ }^{\circ} \mathrm{C}$. The relative velocities of the sphere were respectively $U_{x}=$ $40 \mathrm{~mm} / \mathrm{min}$. and $U_{x}=8 \mathrm{~mm} / \mathrm{min}$. Thus $U_{x} b / 2 v \sim 6 \times 10^{-2}$ and $5 \times 10^{-4}$ in the two cases; the condition of validity of $(1),\left(U_{x} a / v\right) \ll 1$, is also fulfilled. We have checked experimentally that the measurements were done in a stationary regime (constant $F_{x}$ ). The permanent regime establishes itself faster (with a $t^{-3 / 2}$ saturation law) in the presence of a wall than in the absence of it $\left(t^{-1 / 2}\right)[15]$.

The experimental results are presented in figure 1 for the two oils used. For large separations $\left(4 \times 10^{-2}<\varepsilon<0.4\right)$ the results agree with the different theoretical models. We attribute the deviation of experimental points from the theoretical predictions to the effect of the cylindrical wall of the tube $(D \sim 10 \mathrm{~mm})$ which causes additional friction at larger distances from the walls.

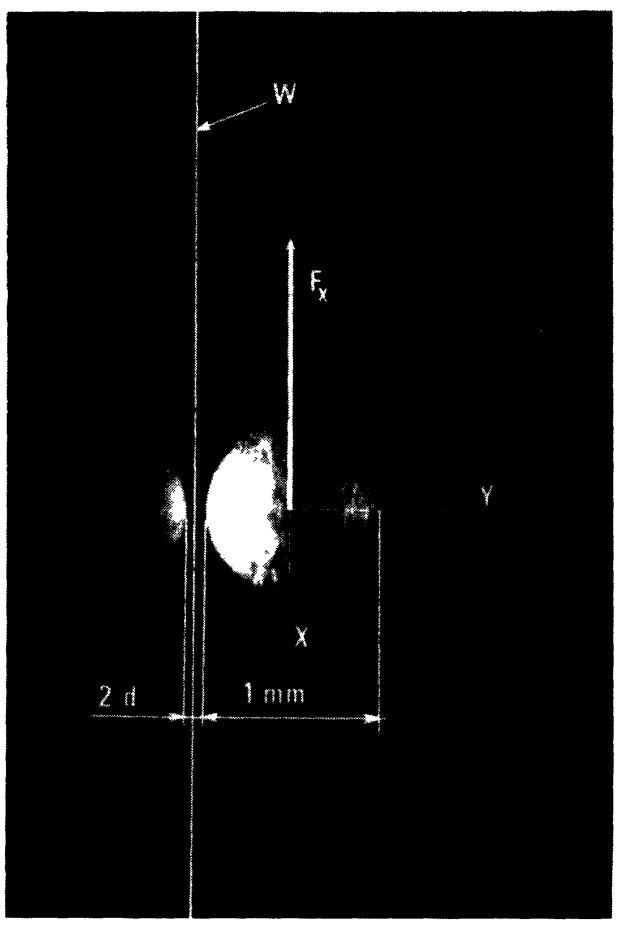

Fig. 2. - Photograph of the magnetic sphere next to the vertical reflecting wall $\mathrm{W}$. The distance $d$ is accurately determined using the reflection image of the sphere, when the tube is displaced vertically. 
Despite the limited accuracy of the measurements of the smallest separations $d$ the results also indicate a saturation of the forces as $d \rightarrow 0$. In O'Neill's solution there is a divergence of $F_{x}$ in the lubrication limit $\varepsilon \rightarrow 0$ which would be due to the friction of the wall and the non rotating sphere in contact with it. On the other hand a saturation effect is predicted in Faxén's model where the sphere is free to rotate near the wall. A similar saturation effect is also found with the model of Goldman et al. [14]. In their treatment they consider separately the effects of translation and rotation, and by superposition they calculate exactly the force in the lubrication limit. When $d \rightarrow 0$, they find that $a \omega / U \sim 1 / 4$. This result is incompatible with the no slip condition at the wall which would imply $a \omega / U=1$. Thus a saturation effect is also expected for small separations.

The present experimental techniques can easily be extended to other projects involving suspensions; like for example measuring the force on a levitated magnetic sphere placed in the middle of an array of spheres falling around it or fixed mechanically and in a moving liquid. Such situations are closer to theoretical models where the velocity fields rather than force fields - as in classical experiments - are defined.

Acknowledgments. - We are very grateful to M. Clément for his helpful assistance. The authors thank C. Deslouis, J. Dufaux, J. P. Jorre, and D. Lhuillier for helpful discussions.

\section{References}

[1] A list of problems to be studied experimentally and theoretically has been proposed by DE GENNES, P. G., Phys. Chem. Hydro. 2 (1981) 31.

[2] Cox, R. G. and Mason, S. G., Ann. Rev. Fluid Mech. 3 (1971) 291.

[3] Gauthier Manuel, B., Meyer, R. and Pieranski, P., Submitted to J. Phys. E.

[4] It is also possible to measure the component $F_{y}$ of the force by measuring the horizontal displacement of the sphere, when the liquid is set in motion, knowing $\mathrm{d} H_{y} / \mathrm{d} y$. This was not done in the present preliminary study.

[5] BRUNN, P., Int. J. Multiphase flow 7 (1981) 221.

[6] Goldsmith, H. L. and Mason, S. G., Rheology, vol. 4 (Acad. Press) 1967, p. 150.

[7] Schultz-Grunow, F., Ing. Archiv. 28 (1959) 307.

[8] Brandt-Bugliarello, Trans. Soc. Rheol. 10 (1966) 229.

[9] LoRENTZ, H. A., Zittingsverlag Akad. Wet. Amsterd. 5 (1896) 168. Abhand-Lungen über theoretisch 1 (1907) S. 23 (Leipzig).

[10] Stock, J., Bull. Int. Acad. Cracovie (1911) A 18-27.

[11] FAXÉN, H., Einwirkung der Gerfäßwände auf den Widerstand gegen die Bewegung einer kleinen Kugel in einer Zähen Flüssigkeit (Diss) (Upsala) (1921) pp. 55-128.

[12] O’NeILL, M. E., Mathematica 11 (1964) 67.

[13] O'Neill, M. E. and Stewartson, K., J. Fluid Mech. 27 (1967) 705.

[14] Goldman, A. J., Cox, R. G., Brenner, M., Chem. Eng. Sci. 22 (1967) 637.

[15] WakiYa, S., J. Phys. Soc. Jpn. 19 (1964) 1401. 